\section{Commentary: Pneumatocele and cysts in a patient with severe acute respiratory syndrome coronavirus-2 (SARS-CoV-2) infection-yet another new complication associated with coronavirus disease 2019 (COVID-19)}

\author{
Danny Ramzy, MD, PhD
}

In December 2019, an outbreak of pneumonia traced to a wet market in Wuhan, China, proliferated into a global pandemic with seemingly exponential vehemence and in just 8 months spread globally, with more than 800,000 deaths. ${ }^{1,2}$ Coronavirus disease 2019 (COVID-19) is caused by the novel betacoronavirus severe acute respiratory syndrome coronavirus-2 (SARS-CoV-2), which is characterized by extreme virulence and a complex spectrum of pathologies ranging in severity from mild constitutional symptoms to multiorgan failure ${ }^{3}$ and death. Over the past 8 months, our knowledge of the pathophysiology and lung parenchymal manifestations has grown tremendously, yet despite this we are constantly discovering new manifestations and new pathologies associated with this deadly virus. With most of the literature currently focused on treatment strategies and symptom recognition, it's easy to forget that unique and potentially fatal complications are yet to be identified.

In the current issue of Journal, Brahmbhatt and colleagues ${ }^{4}$ provide a single case report describing a newly

\footnotetext{
From the Department of Cardiac Surgery, Cedars-Sinai Medical Center, Los Angeles, Calif.

Disclosures: The author reported no conflicts of interest.

The Journal policy requires editors and reviewers to disclose conflicts of interest and to decline handling or reviewing manuscripts for which they may have a conflict of interest. The editors and reviewers of this article have no conflicts of interest.

Received for publication Aug 28, 2020; revisions received Aug 28, 2020; accepted for publication Sept 5, 2020; available ahead of print Sept 15, 2020.

Address for reprints: Danny Ramzy, MD, PhD, Cedars-Sinai Medical Center, $127 \mathrm{~S}$. San Vicente Blvd, Suite A3105, Los Angeles, CA (E-mail: danny.ramzy @ cshs.org). JTCVS Techniques 2020;4:358-9

2666-2507

Copyright $(2020$ The Authors. Published by Elsevier Inc. on behalf of The American Association for Thoracic Surgery. This is an open access article under the CC BY-NCND license (http://creativecommons.org/licenses/by-nc-nd/4.0/).

https://doi.org/10.1016/j.xjtc.2020.09.012
}

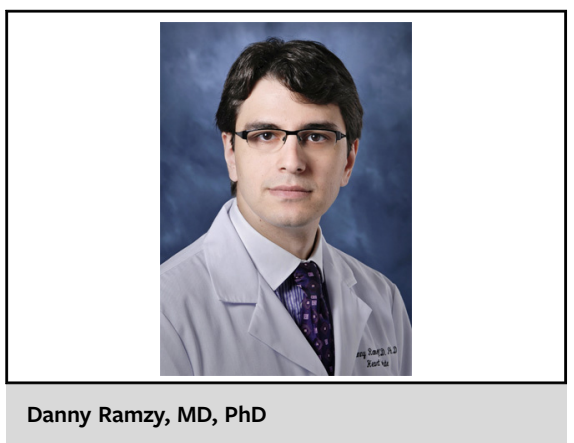

CENTRAL MESSAGE

COVID-19 and its parenchymal changes have been documented. However, this case report demonstrates that while we have obtained a greater understanding, we still have more to learn about this virus.

discovered, potential life-threatening complication, more specifically, pneumatocele and cyst formation in a patient following SARS-CoV2 infection with acute respiratory distress syndrome (ARDS). This is the first such report of this complication. Brahmbhatt and colleagues ${ }^{4}$ provide a detailed case report beginning with diagnosis and proceeding to the corrective clinical steps. Our cardiothoracic surgery community has become accustomed to equating COVID-19 computed tomography image findings as bilateral, peripheral/subpleural, or posterior ground-glass opacities with or without consolidations. The global community is rapidly realizing that the manifestations of COVID-19 infection have yet to be fully elucidated. To illustrate this point, Brahmbhatt and colleagues ${ }^{4}$ describe their case of a 66-year-old female patient with SARS-CoV2 ARDS resulting in novel COVID-19-related complications. Their patient had several known high-risk factors for poor outcome with a course typical of those manifesting a rapid decline postinfection. With a rapid decline in oxygenation, treatment with hydroxychloroquine was initiated. We now know that hydroxychloroquine has no benefit and may in fact increase mortality. This highlights the rapid evolution of our knowledge of COVID-19 while reminding us that we have a great deal more to learn about this disease.

As described by Brahmbhatt and colleagues, ${ }^{4}$ COVID-19 can result in significant lung injury leading to substantial morbidity. Brahmbhatt and colleagues' manuscript 
highlights a novel finding in the presence of pneumatoceles. The development of spontaneous pneumothoraces are just starting to be reported. ${ }^{5,6}$ Brahmbhatt and colleagues' finding suggests that a substantial percentage of COVID19 pneumothoraces may actually be as of a result of cystic or pneumatocele rupture. Their team treated the pneumatoceles with computed tomography-guided pig-tail insertion. This may not the first choice of treatment, however, as pneumatoceles are usually treated by observation alone. While observation works in a majority of cases, it may not be appropriate in patients with COVID-19, especially with the high likelihood of respiratory and oxygenation status deterioration. Given the current recommendation to avoid intubating and placing patients with COVID-19 on ventilators to reduce lung injury-especially in a patient with a pneumatoceles-treatment with percutaneous drainage was the more appropriate and wiser choice. Their case also illustrates one additional mechanism through which intubating and mechanically ventilating patients with COVID-19 results in worsening clinical and radiologic status. While Brahmbhatt and colleagues ${ }^{4}$ effectively treated the pneumatoceles and the patient, the concern that I raise is the need for a national standardized approach and treatment protocols for SARS-CoV2 ARDS. However, the most vital conclusion is that we are still learning and discovering new manifestations of COVID-19. If we lose sight of this valuable lesson, our patients may ultimately pay the price in morbidity and mortality. The manuscript by
Brahmbhatt and colleagues ${ }^{4}$ is yet another reminder of the importance of the cardiac surgical team remaining hypervigilant in identifying novel, as well as known, manifestations and complications of COVID-19 before they become life-threatening.

The aggressive spread and multifaceted evolution of COVID-19 mandates that the cardiac surgical community understand the effects of COVID-19 and remain vigilant toward undiscovered manifestations to guide future treatment strategies. As the disease continues to proliferate across all continents, Brahmbhatt and colleagues' findings are yet another reminder that we must remain hypervigilant at identifying new manifestations and improving our treatment strategies.

\section{References}

1. Zhu N, Zhang D, Wang W, Li X, Yang B, Song J, et al. A novel coronavirus from patients with pneumonia in China, 2019. N Engl J Med. 2020;382:727-33.

2. Wu Y-C, Chen C-S, Chan Y-J. The outbreak of COVID-19: an overview. J Chin Med Assoc. 2020;83:217-20.

3. Siddiqi HK, Mehra MR. COVID-19 illness in native and immunosuppressed states: a clinical-therapeutic staging proposal. J Heart Lung Transplant. 2020; 39:405-7.

4. Brahmbhatt N, Tamimi O, Ellison H, Katta S, Youssef J, Cortes C, et al Pneumatocele and cysts in a patient with severe acute respiratory syndrome coronavirus 2 infection. J Thorac Cardiovasc Surg Tech. 2020;4:353-5.

5. Khurram R, Johnson FTF, Naran R, Hare S. Spontaneous tension pneumothorax and acute pulmonary emboli in a patient with COVID-19 infection. BMJ Case Rep. 2020;13:e237475.

6. Mallick T, Dinesh A, Engdahl R, Sabado M. COVID-19 complicated by spontaneous pneumothorax. Cureus. 2020;12:e9104. 